\title{
A diffusion-controlled regime of cylindrical inductive discharges
}

\author{
St Kolev ${ }^{1}$, H Schlüter ${ }^{2}$, A Shivarova ${ }^{1}$ and Kh Tarnev ${ }^{3}$ \\ ${ }^{1}$ Faculty of Physics, Sofia University, BG-1164 Sofia, Bulgaria \\ ${ }^{2}$ Experimental Physics 2, Ruhr-University Bochum, D-44780 Bochum, Germany \\ ${ }^{3}$ Department of Applied Physics, Technical University-Sofia, BG-1000 Sofia, Bulgaria
}

Received 20 April 2006, in final form 25 July 2006

Published 22 August 2006

Online at stacks.iop.org/PSST/15/744

\begin{abstract}
A fluid-plasma model of diffusion-controlled cylindrical inductive discharges in an argon gas is presented. The plasma-field structure of the discharge obtained is completed by the interrelated behaviour of concentrations of charged particles, electron temperature, power absorbed on average by an electron, radial distribution of the components of the high-frequency field, of the Joule heating and of the high-frequency current density in the plasma. The self-consistency of the model and its validity over a wide pressure range ( $p=(0.05-5)$ Torr) is reached by involving detailed treatment of the electron energy balance, of the nonlinear processes in the charged particle balance and of the momentum equations. By accounting for the velocity dependence of the elastic electron-neutral collision frequency, concepts from the kinetic plasma theory are introduced in a fluid-plasma description of the discharge. The analysis of the results is in terms of changing gas pressure, power and frequency of the maintenance field. The changes of the parameters of the external coil due to the plasma loading in the coil are also discussed.
\end{abstract}

\section{Introduction}

The wide use of inductive discharges in plasma processing technology $[1,2]$ has motivated their active study over the years [3-8]. Besides the discharge models based on the kinetic plasma theory [9-14], aiming at determination of the electron energy distribution function in the discharge and specifying the electron heating at low gas pressures, the theoretical description of the discharge has been developed in two directions. The first one is the so-called transformer model $[1,6,8,15-18]$ in which the information for the plasma parameters comes out from the description of the electrical characteristics of an equivalent transformer coupled circuit completed by the external coil (as a primary circuit) and the plasma (as a secondary one). Fluid plasma models [6-8] of the discharge combining electrodynamics with gasdischarge description form the second direction in discharge modelling.

The diffusion-controlled regime of maintenance-in a wide pressure range - of inductive discharges in an argon gas is treated here. The study presents a self-consistent 1D fluid-plasma model of the discharge developed by involving recent achievements in modelling of surface-wave-sustained discharges [19-26], inductive discharges [8,27, 28] and highfrequency discharges (e.g. [29]), in general.

Gas-discharge description and description of the electromagnetic field producing the discharge are coupled selfconsistently in the model presented here. The gas-discharge part of the model is based on the concept [25] of the generalized Schottky condition for discharges in atomic gases which states that the total losses (by ambipolar diffusion and recombination) are compensated by the total ionization (direct and step ionization). The Schottky condition, still widely used in the fluidplasma models of the inductive discharges, does not provide a self-consistent description because it equalizes only direct ionization to the diffusion losses. The nonlinear processes of step ionization and recombination-in the charged particle balance-ensure, in general, the coupling of the plasma density $n_{\mathrm{e}}$ to the electron temperature $T_{\mathrm{e}}$ (or, equivalently, to the power $\Theta$ absorbed on average by one electron) and thus, the discharge self-consistency. Moreover, accounting for the step ionization is a requisite for a proper description of the inductive discharges because of the high-density plasma production in these discharges. 
When the high-pressure range of the diffusion-controlled regime is aimed to be also covered by the model, like here, the recombination should be also taken into account. This motivates the detailed description of the dissociative recombination (DR) included in the model. Description of the ion dynamics, with effective mobilities and effective ambipolar diffusion coefficients introduced, extends the model towards the low-pressure range of the diffusion-controlled discharges and, partially, to the transition to the free-fall regime. For proper covering of the wide pressure range considered, the electron energy balance is also treated precisely. The electron energy balance includes not only losses through thermal conductivity and collisions (inelastic and elastic) but also fluxes of thermal energy and pressure-force work carried out by the directed motion as well as losses for maintaining the ambipolar field.

Accounting for the velocity dependence of the electronneutral elastic collision frequency requires using electron transport coefficients (mobility and diffusion coefficients for momentum transfer) derived from the Boltzmann equation for inhomogeneous plasmas in high-frequency and dc fields (respectively, the field sustaining the discharge and the ambipolar field). This not only involves in the fluidplasma description the integral representation of the plasma conductivity $[27,28,30,31]$ (and, respectively, of the plasma permittivity used in the electrodynamical part of the model) but also modifies the electron energy balance.

The electrodynamical part of the model provides a description of the electromagnetic field in radiallyinhomogeneous plasmas, with a radial profile of the plasma density - a Bessel type of profile-as derived in the gas discharge part of the model. Based on the radial field distribution stemming from the electrodynamical part of the model, the current density in the plasma and the Joule heating are obtained and discussed with respect to the power deposition in the discharge. Comparison with results obtained by taking an averaged plasma density in the electrodynamical part of the model shows the necessity of accounting for the radial plasma density inhomogeneity. The changes of the parameters of the external coil due to the plasma loading in the coil are also commented on.

The discussions on the self-consistent discharge structure concern the modifications of the discharge characteristics with varying gas pressure, power and frequency of the maintenance field.

\section{Basis of the model}

An inductive discharge with a cylindrical configuration maintained in a diffusion controlled regime in an argon gas is considered. The discharge is produced by using an external coil of $N$ turns and a length $L$ positioned over the gas-discharge tube (figure 1). The internal and external radii of the tube are, respectively, $R$ and $R^{\prime}$. As it is known [1], the maintenance of the inductive discharges is by an azimuthal electric field $\left(E_{\varphi}\right)$ induced by the axial magnetic field $\left(H_{z}\right)$ which is produced by the high-frequency current in the coil. In fact, a transverse high-frequency wave-of frequency $\omega$, propagation constant in a radial direction and field components $E_{\varphi}$ and $H_{z}$-which penetrates into the plasma over the distance of the skin depth

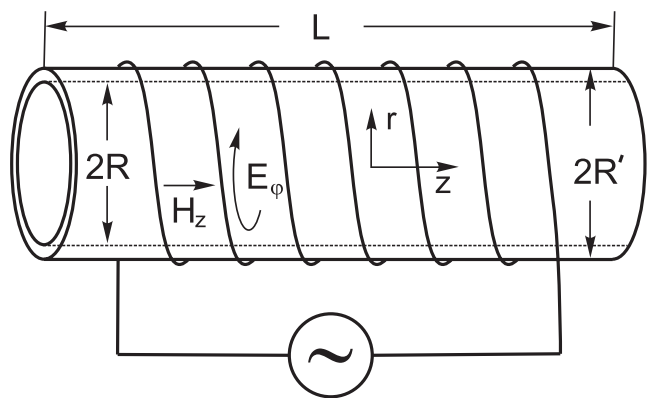

Figure 1. A schematical representation of the discharge.

sustains the discharge. With a length of the coil $L>R$, the edge effects are neglected and the description of the discharge is within an 1D model.

Electrons, atomic $\left(\mathrm{Ar}^{+}\right)$and molecular $\left(\mathrm{Ar}_{2}^{+}\right)$ions as well as ground-state and excited atoms are the species in the discharge. The balance of the molecular ions is included regarding the DR treated in the model. The metastable argon states considered as a block are taken into account in the description of both the step ionization and the DR. Similar simplified atomic models including only metastable states or the first four excited states, also as a block, have been often employed $[14,20,23,32,33]$ in description of main trends of the discharge behaviour. The gas pressure $p=N_{0} \kappa T_{\mathrm{g}}$, where $N_{0}$ is the ground-state neutral density and $\kappa$ is the Boltzmann constant, as well as the gas temperature $T_{\mathrm{g}}$ and the total applied power $P$ are external parameters.

The gas-discharge part of the model is based on

(i) the continuity equations

$$
\operatorname{div}\left(n_{\alpha} \boldsymbol{u}_{\alpha}\right)=\frac{\delta n_{\alpha}}{\delta t}
$$

of the charged particles (where $\alpha=e, 1,2$ for electrons and for the two types of ions $\mathrm{Ar}^{+}$and $\mathrm{Ar}_{2}^{+}$-ions denoted, respectively, by ' 1 ' and '2'),

(ii) their momentum equations and

(iii) the electron-energy balance equation

$$
-\operatorname{div} \boldsymbol{J}_{\mathrm{e}}-e n_{\mathrm{e}} \boldsymbol{u}_{\mathrm{e}} \cdot \boldsymbol{E}+\frac{\delta\left(n\left\langle K_{\mathrm{e}}\right\rangle\right)}{\delta t}=0 .
$$

In (1) and (2), $n_{\alpha}$ and $\boldsymbol{u}_{\alpha}$ are, respectively, densities and directed velocities, $\delta n_{\alpha} / \delta t$ represents the particle production and losses through collisions, $\boldsymbol{J}_{\mathrm{e}}$ is the electron-energy flux and $\delta\left(n\left\langle K_{\mathrm{e}}\right\rangle\right) / \delta t$ describes the electron-energy losses in collisions. The electric field $\boldsymbol{E}$ in (2) is the total electric field

$$
\boldsymbol{E}=\overline{\boldsymbol{E}}_{\mathrm{A}}+\frac{1}{2}\left\{\tilde{\boldsymbol{E}} \mathrm{e}^{-\mathrm{i} \omega t}+\text { c.c. }\right\}
$$

including both the high-frequency field $\tilde{\boldsymbol{E}}$ sustaining the discharge and the dc ambipolar field $\overline{\boldsymbol{E}}_{A}$.

The electrodynamical part of the model is based on the wave equation written in its form

$$
\Delta \boldsymbol{H}+\frac{1}{\varepsilon(\omega, \boldsymbol{r})} \operatorname{grad} \varepsilon(\omega, \boldsymbol{r}) \times \operatorname{curl} \boldsymbol{H}+\frac{\omega^{2} \varepsilon(\omega, \boldsymbol{r})}{c^{2}} \boldsymbol{H}=0
$$

for the magnetic field of the wave in inhomogeneous plasma with permittivity $\varepsilon(\omega, \boldsymbol{r}) ; c$ is the light speed in vacuum. The gas-discharge and electrodynamical descriptions are 
coupled not only through the plasma density and, in general, through the conductivity which determines $\varepsilon(\omega, \boldsymbol{r})$ but also through the total absorbed power $Q_{\text {total }}=P$. In the gasdischarge part of the model the latter is given by expression

$$
Q_{\text {total }} \equiv \pi R^{2} L \bar{n}_{\mathrm{e}} \Theta=P
$$

where $\bar{n}_{\mathrm{e}}$ and $\Theta$ are, respectively, the averaged-over the discharge cross section-plasma density and power absorbed by an electron, whereas in the electrodynamical part of the model the corresponding expression is

$$
Q_{\text {total }} \equiv \frac{1}{2} \int_{(V)}\left(\operatorname{Re} \sigma_{\tilde{E}}\right)\left|E_{\varphi}\right|^{2} \mathrm{~d} V=P
$$

$\operatorname{Re} \sigma_{\tilde{E}}$ is the real part of the plasma conductivity.

The self-consistent plasma-field structure of the discharge obtained from (1)-(5a) and (5b) is built by the interrelated behaviour of concentrations of charged particles, electron temperature, power $\Theta$ and radial distribution of the components of the high-frequency field, as given in sections 3 and 4. The skin depth as well as the Joule heating and the high-frequency current in the plasma (section 4) are the other important characteristics of the discharge determined by the above-listed quantities.

\section{Gas-discharge part of the model}

The final set of equations of the gas-discharge part of the model includes the balance equations of the $\mathrm{Ar}^{+}$- and $\mathrm{Ar}_{2}^{+}$-ions $\left(n_{1}\right.$ and $n_{2}$ are the corresponding concentrations) completed with the balance of the excited atoms (with concentration $N_{j}$ ), the condition for quasi-neutrality, the electron energy balance, the Bohm criterion at the discharge walls and expression $(5 a)$ for the total high-frequency power.

\subsection{Charged particle balance}

For solving the continuity equations (1) and completing the particle balance, the directed velocity $\boldsymbol{u}_{\alpha}$ of the charged particles and the processes for particle production and losses through collisions, summarized in the terms $\left(\delta n_{\alpha} / \delta t\right)$ in (1), should be first specified.

3.1.1. Mobility and diffusion coefficients. Accounting for the velocity dependence of the elastic electrons-neutral collision frequency $v_{\mathrm{e}-\mathrm{n}}$ requires determination of the directed velocity of the electrons $\boldsymbol{u}_{\mathrm{e}}$ from the anisotropic part $f_{1}(v)$ of the electron velocity distribution function $f(v)=f_{0}(v)+(v / v)$. $\boldsymbol{f}_{1}(v)$ obtained from the Boltzmann equation [30,31]. With an electric field including both dc $\left(\overline{\boldsymbol{E}}_{\mathrm{A}}\right)$ and ac $(\tilde{\boldsymbol{E}})$ fields, as given by (3), the solution for $f_{1}$, obtained in the same form, results in the following expression for the directed velocity of the electrons:

$$
\begin{aligned}
\boldsymbol{u}_{\mathrm{e}}= & \frac{4 \pi}{3}\left[\frac{e \overline{\boldsymbol{E}}_{\mathrm{A}}}{m_{\mathrm{e}}} \int_{0}^{\infty} \frac{v^{3}}{v_{\mathrm{e}-\mathrm{n}}} \frac{\mathrm{d} f_{0}}{\mathrm{~d} v} \mathrm{~d} v-\frac{1}{n_{\mathrm{e}}} \int_{0}^{\infty} \frac{v^{4}}{v_{\mathrm{e}-\mathrm{n}}} \nabla\left(n_{\mathrm{e}} f_{0}\right) \mathrm{d} v\right] \\
& +\frac{4 \pi}{6}\left[\frac{e \tilde{\boldsymbol{E}}}{m_{\mathrm{e}}} \mathrm{e}^{-\mathrm{i} \omega t} \int_{0}^{\infty} \frac{v^{3}}{-\mathrm{i} \omega+v_{\mathrm{e}-\mathrm{n}}} \frac{\mathrm{d} f_{0}}{\mathrm{~d} v} \mathrm{~d} v+\text { c.c. }\right]
\end{aligned}
$$

Here $e$ and $m_{\mathrm{e}}$ are, respectively, the electron charge and mass; $f_{0}(v)$ is the isotropic part of the distribution function taken as a Maxwellian one.

Comparison of the stationary (time-averaged) part of (6) with the fluid-model momentum equation for the electrons

$$
n_{\mathrm{e}} \overline{\boldsymbol{u}}_{\mathrm{e}}=-b_{\mathrm{e}} n_{\mathrm{e}} \overline{\boldsymbol{E}}_{\mathrm{A}}-\nabla\left(D_{\mathrm{e}} n_{\mathrm{e}}\right)
$$

defines the electron mobility $b_{\mathrm{e}}$ and the electron diffusion coefficient:

$$
\begin{gathered}
b_{\mathrm{e}}=-\frac{4 \pi e}{3 m_{\mathrm{e}}} \int_{0}^{\infty} \frac{v^{3}}{v_{\mathrm{e}-\mathrm{n}}} \frac{\mathrm{d} f_{0}}{\mathrm{~d} v} \mathrm{~d} v, \\
D_{\mathrm{e}}=\frac{4 \pi}{3} \int_{0}^{\infty} \frac{v^{4}}{v_{\mathrm{e}-\mathrm{n}}} f_{0} \mathrm{~d} v .
\end{gathered}
$$

The directed motion of the ions is only in the dc field $\overline{\boldsymbol{E}}_{\mathrm{A}}$. Their velocities are obtained from the ordinary form of the stationary momentum equation [31], however, with both elastic and inelastic collisions taken into account:

$$
\boldsymbol{u}_{\alpha}=b_{\alpha} \overline{\boldsymbol{E}}_{\mathrm{A}}-\frac{D_{\alpha} \nabla n_{\alpha}}{n_{\alpha}},
$$

where

$$
\begin{aligned}
& b_{\alpha}=\frac{e}{\mu_{\alpha-n} v_{\alpha-n}+m_{\alpha}\left(1 / n_{\alpha}\right)\left(\delta n_{\alpha} / \delta t\right)}, \\
& D_{\alpha}=\frac{T_{i \alpha}}{\mu_{\alpha-n} v_{\alpha-n}+m_{\alpha}\left(1 / n_{\alpha}\right)\left(\delta n_{\alpha} / \delta t\right)}
\end{aligned}
$$

are the ion mobility and diffusion coefficients. Here $\alpha=1,2$ for the two types of ions ( $\mathrm{Ar}^{+}$and $\mathrm{Ar}_{2}^{+}$, respectively), $m_{\alpha}$ and $\mu_{\alpha-n}$ are, respectively, the masses of the ions and their reduced masses and $v_{\alpha-n}$ are the ion-neutral elastic collision frequencies.

The equality of the electron- and ion-fluxes, with accounting that $b_{\mathrm{e}} \gg b_{1,2}$ and $T_{\mathrm{e}} \gg T_{i 1,2}$ hold for the mobilities and the temperatures of electrons and ions, results in the final expression for the charged particle velocities in the ambipolar field:

$$
\begin{aligned}
\overline{\boldsymbol{u}}_{\mathrm{e}} & =-D_{\mathrm{A} e} \frac{\nabla n_{\mathrm{e}}}{n_{\mathrm{e}}}, \\
\boldsymbol{u}_{1,2} & =-D_{\mathrm{A} 1,2} \frac{\nabla n_{\mathrm{e}}}{n_{\mathrm{e}}},
\end{aligned}
$$

where

$$
\begin{gathered}
D_{\mathrm{A} 1,2}=\frac{T_{\mathrm{e}}}{e} b_{1,2}, \\
D_{\mathrm{A} e}=\frac{1}{n_{\mathrm{e}}}\left(D_{\mathrm{A} 1} n_{1}+D_{\mathrm{A} 2} n_{2}\right)
\end{gathered}
$$

are, respectively, the ambipolar diffusion coefficients of ions and electrons. Using effective mobilities $(8 b)$ of the ions, which account for the inelastic collisions, and defining, respectively, effective ambipolar diffusion coefficients (10a) extend the validity of the model towards lower pressures. The electron velocity $(9 a)$ expressed through the ambipolar diffusion coefficient $(10 b)$ is not influenced by the velocity dependence of the elastic electron-neutral collision frequency. 
Table 1. Processes included in the particle balance. In the table, $T_{\mathrm{e}}$ is in $[\mathrm{eV}]$ and $T_{\mathrm{g}}$ in $[\mathrm{K}] ; g_{0}$ and $g_{j}$ are the corresponding statistical weights and $U_{i}$ and $U_{j}$ are, respectively, the energies of ionization and excitation of the metastable state block.

\begin{tabular}{llll}
\hline No & Process & Rate coefficients & References \\
\hline 1. & $\mathrm{Ar}+\mathrm{e}^{-} \rightarrow \mathrm{Ar}^{+}+2 \mathrm{e}^{-}$ & $k_{i\left(\mathrm{~m}^{3} / \mathrm{s}\right)}=1.41 \times 10^{-14} T_{\mathrm{e}}^{0.69} \exp \left(-\frac{U_{i}}{T_{\mathrm{e}}}\right)$ & {$[35]$} \\
2. & $\mathrm{Ar}+\mathrm{e}^{-} \rightarrow \mathrm{Ar}^{*}+\mathrm{e}^{-}$ & $k_{0 j}$ : polynomial representation & {$[36]$} \\
3. & $\mathrm{Ar}^{*}+\mathrm{e}^{-} \rightarrow \mathrm{Ar}^{+}+2 \mathrm{e}^{-}$ & $k_{j i}:$ polynomial representation & {$[37]$} \\
4. & $\mathrm{Ar}^{*}+\mathrm{e}^{-} \rightarrow \mathrm{Ar}+\mathrm{e}^{-}$ & $k_{j 0\left(\mathrm{~m}^{3} / \mathrm{s}\right)}=k_{0 j}\left(g_{0} / g_{j}\right) \exp \left(U_{j} / T_{\mathrm{e}}\right)$ & {$[38]$} \\
5. & $\mathrm{Ar}^{+}+2 \mathrm{Ar} \rightarrow \mathrm{Ar}_{2}^{+}+\mathrm{Ar}$ & $k_{\mathrm{AAA}\left(\mathrm{m}^{6} / \mathrm{s}\right)}=2.5 \times 10^{-43}\left(T_{\mathrm{g}} / 300\right)^{-3 / 2}$ & {$[39]$} \\
6. & $\mathrm{Ar}^{*}+\mathrm{Ar}^{*} \rightarrow \mathrm{Ar}_{2}^{+}+\mathrm{e}^{-}$ & $k_{\mathrm{MAI}\left(\mathrm{m}^{3} / \mathrm{s}\right)}=6.3 \times 10^{-16}\left(T_{\mathrm{g}} / 300\right)^{-1 / 2}$ & {$[39]$} \\
7. & $\mathrm{Ar}_{2}^{+}+\mathrm{e}^{-} \rightarrow \mathrm{Ar}+\mathrm{Ar}^{*}$ & $k_{\mathrm{DR} 1\left(\mathrm{~m}^{3} / \mathrm{s}\right)}=7.35 \times 10^{-14} T_{\mathrm{e}}^{-0.67} h_{1}$ & {$[34,40]$} \\
8. & $\mathrm{Ar}_{2}^{+}+\mathrm{e}^{-} \rightarrow \mathrm{Ar}+\mathrm{Ar}$ & $k_{\mathrm{DR} 2\left(\mathrm{~m}^{3} / \mathrm{s}\right)}=7.35 \times 10^{-14} T_{\mathrm{e}}^{-0.67} h_{2}$ & {$[34,40]$} \\
9. & $\mathrm{Ar}_{2}^{+}+\mathrm{e}^{-} \rightarrow \mathrm{Ar}^{+}+\mathrm{Ar}+\mathrm{e}^{-}$ & $k_{\mathrm{DEI}\left(\mathrm{m}^{3} / \mathrm{s}\right)}=1.11 \times 10^{-12} \exp \left(-2.94 / T_{\mathrm{e}}\right)$ & {$[34,39]$} \\
10. & $\mathrm{Ar}_{2}^{+}+\mathrm{Ar} \rightarrow \mathrm{Ar}^{+}+2 \mathrm{Ar}$ & $k_{\mathrm{DAI}\left(\mathrm{m}^{3} / \mathrm{s}\right)}=\frac{6.06 \times 10^{-12}}{T_{\mathrm{g}}} \exp \left(-\frac{1.51 \times 10^{4}}{T_{\mathrm{g}}}\right)$ & {$[34]$} \\
11. & $\mathrm{Ar}^{+}+2 \mathrm{e}^{-} \rightarrow \mathrm{Ar}+\mathrm{e}^{-}$ & $k_{\mathrm{TR}\left(\mathrm{m}^{6} / \mathrm{s}\right)}=2.6 \times 10^{-39} T_{\mathrm{e}}^{-4.5}$ & {$[38]$} \\
\hline
\end{tabular}

3.1.2. Particle production and losses through collisions. The particle production and losses through collisions are according to the processes listed in table 1. The argon atom ionization is via direct and step ionization (reactions (1) and (3) in table 1). The balance of the $\mathrm{Ar}_{2}^{+}$-ions [34] includes their production by atom assisted association of atoms and ions (AAA, reaction 5) and metastable-metastable associative ionization (MAI, reaction 6) and losses by DR (reactions 7 and 8 ) and dissociation by electron and atom impact (reactions 9 and 10, respectively, with notation DEI and DAI). Three body recombination of $\mathrm{Ar}^{+}$-ions (reaction 11) is also included in the electron- and $\mathrm{Ar}^{+}$-ion balance. The rate coefficients for the direct and step ionization as well as for excitation are obtained by numerical integration of the cross sections over a Maxwellian electron energy distribution. The data for these cross sections as well as the rate coefficients for the processes involved in the production and destruction of the molecular ions are taken from [34-40] as given in table 1 . In the coefficients $k_{\mathrm{DR} 1}$ and $k_{\mathrm{DR} 2}$ the efficiencyaccording to [34] - of the corresponding process is also included.

3.1.3. Final set of equations for the particle balance. The radial profile of the electron concentration

$$
n_{\mathrm{e}}(r)=n_{\mathrm{e}}(r=0) J_{0}\left(\frac{\mu r}{R}\right)
$$

is a Bessel type of profile, as expected for diffusion controlled discharges provided the following assumptions are made in the balance equations of the $\mathrm{Ar}^{+}$- and $\mathrm{Ar}_{2}^{+}$-ions (with densities $n_{1}$ and $n_{2}$, respectively): (i) radially independent ratios $n_{1,2} / n_{\mathrm{e}}$ and (ii) a linearization of the nonlinear terms by replacements of the type of $n_{2}(r) n_{\mathrm{e}}(r) \rightarrow n_{2}(r=0) n_{\mathrm{e}}(r)$. The former is in agreement with the condition of quasi-neutrality

$$
n_{\mathrm{e}}=n_{1}+n_{2}
$$

and the latter has been widely used before [20-26] in models of surface-wave-sustained discharges, after the detailed check of its validity in [19]. The balance equations of the two types of positive ions obtained from (1) after using (9a), (11) and table 1 are as follows:

$$
\begin{aligned}
& \left(\frac{\mu}{R}\right)^{2} D_{\mathrm{A} 1} n_{1}(r=0)=\left[k_{i} N_{0}+k_{j i} N_{j}(r=0)+k_{\mathrm{DEI}} n_{2}(r=0)\right. \\
& \left.\quad-k_{\mathrm{TR}} n_{1}(r=0) n_{\mathrm{e}}(r=0)\right] n_{\mathrm{e}}(r=0) \\
& \quad+k_{\mathrm{DAI}} N_{0} n_{2}(r=0)-k_{\mathrm{AAA}} N_{0}^{2} n_{1}(r=0) \\
& \left(\frac{\mu}{R}\right)^{2} D_{\mathrm{A} 2} n_{2}(r=0)=k_{\mathrm{AAA}} N_{0}^{2} n_{1}(r=0)+k_{\mathrm{MAI}} N_{j}^{2}(r=0) \\
& \quad-\left(k_{\mathrm{DR} 1}+k_{\mathrm{DR} 2}+k_{\mathrm{DEI}}\right) n_{2}(r=0) n_{\mathrm{e}}(r=0) \\
& \quad-k_{\mathrm{DAI}} N_{0} n_{2}(r=0)
\end{aligned}
$$

The parameter $\mu$ of the radial plasma-density inhomogeneity is obtained by applying the Bohm criterion for plasmas with two types of positive ions [41]:

$$
\sum_{k=1,2} \frac{e^{2} n_{k}(r=0)}{m_{\mathrm{i} k} \mathrm{u}_{k}^{2}(r=R)-T_{\mathrm{i}}(r=R)}=\frac{e^{2} n_{\mathrm{e}}(r=0)}{T_{\mathrm{e}}(r=R)} .
$$

The particle balance (equations (13) and (14) for the ions) is completed by including the balance equation also of the excited atoms:

$$
\begin{aligned}
& {\left[\frac{D_{j}}{\Lambda_{j}^{2}}+k_{j i} n_{\mathrm{e}}(r=0)+k_{j 0} n_{\mathrm{e}}(r=0)\right] N_{j}(r=0)} \\
& +k_{\mathrm{MAI}} N_{j}^{2}(r=0) \\
& =\left[k_{0 j} N_{0}+k_{\mathrm{DR} 1} n_{2}(r=0)\right] n_{\mathrm{e}}(r=0),
\end{aligned}
$$

where $D_{j}$ and $\Lambda_{j}$ are the corresponding diffusion coefficient and diffusion length.

\subsection{Electron energy balance}

The velocity dependence of the elastic electron-neutral collision frequency influences the electron energy balance through the electron energy flux $\boldsymbol{J}_{\mathrm{e}}$ (in the first term in (2)) and the Joule heating of the electrons (included in the second term in (2)), thus requiring involvement of the kinetic plasma-model description as it has been proceeded in obtaining (6). 
Comparison of the kinetic plasma-model representation of $\boldsymbol{J}_{\mathrm{e}}$ :

$$
\boldsymbol{J}_{\mathrm{e}}=-b_{\varepsilon} n_{\mathrm{e}} \overline{\boldsymbol{E}}_{\mathrm{A}}-\operatorname{grad}\left(D_{\varepsilon} n_{\mathrm{e}}\right)
$$

with

$$
\begin{gathered}
b_{\varepsilon}=-\frac{2 \pi e}{3} \int_{0}^{\infty} \frac{v^{5}}{v_{\mathrm{e}-\mathrm{n}}} \frac{\mathrm{d} f_{0}}{\mathrm{~d} v} \mathrm{~d} v, \\
D_{\varepsilon}=\frac{2 \pi m_{\mathrm{e}}}{3} \int_{0}^{\infty} \frac{v^{6}}{v_{\mathrm{e}-\mathrm{n}}} f_{0} \mathrm{~d} v
\end{gathered}
$$

being the mobility and the diffusion coefficient for energy transfer, with its fluid-plasma model representation

$$
\boldsymbol{J}_{\mathrm{e}}=\boldsymbol{q}_{\mathrm{e}}+\frac{5}{2} n_{\mathrm{e}} T_{\mathrm{e}} \overline{\boldsymbol{u}}_{\mathrm{e}}
$$

which includes not only thermal conductivity flux $\boldsymbol{q}_{\mathrm{e}}$ but also energy of thermal electron motion and pressure-force work carried by the directed velocity $\overline{\boldsymbol{u}}_{\mathrm{e}}$ (the latter given by (7a) $(7 b)$ and $(7 c)$ ), results in the final form

$$
\boldsymbol{J}_{\mathrm{e}}=-\left(\frac{5}{2}-g_{u}\right) n_{\mathrm{e}} D_{\mathrm{e}} \operatorname{grad} T_{\mathrm{e}}+\left(\frac{5}{2}-g_{u}\right) n_{\mathrm{e}} T_{\mathrm{e}} \overline{\boldsymbol{u}}_{\mathrm{e}}
$$

of the electron-energy flux in the fluid plasma-model description. The quantity $g_{u}=-T_{\mathrm{e}} v_{\mathrm{e}-\mathrm{n}}\left(\partial v_{\mathrm{e}-\mathrm{n}}^{-1} / \partial T_{\mathrm{e}}\right)$ is that involved due to velocity dependence of $v_{\mathrm{e}-\mathrm{n}}$.

The second term in the electron energy balance (2),

$$
-e n_{\mathrm{e}} \boldsymbol{u}_{\mathrm{e}} \cdot \boldsymbol{E}=\frac{1}{2}\left(\operatorname{Re} \sigma_{\tilde{\boldsymbol{E}}}\right)|\tilde{\boldsymbol{E}}|^{2}-e n_{\mathrm{e}} \overline{\boldsymbol{E}}_{\mathrm{A}} \cdot \overline{\boldsymbol{u}}_{\mathrm{e}}
$$

is obtained using (3) and (6). The high-frequency plasma conductivity

$$
\sigma_{\tilde{\boldsymbol{E}}}=-\frac{4 \pi}{3} \frac{e^{2} n_{\mathrm{e}}}{m_{\mathrm{e}}} \int_{0}^{\infty} \frac{v^{3}}{-\mathrm{i} \omega+v_{\mathrm{e}-\mathrm{n}}} \frac{\mathrm{d} f_{0}}{\mathrm{~d} v} \mathrm{~d} v
$$

included in the Joule heating - the first term in the right-hand side of (20) - accounts for the velocity dependence of $v_{\mathrm{e}-\mathrm{n}}$. The second term therein, with $\overline{\boldsymbol{E}}_{\mathrm{A}} \cong-\left(T_{\mathrm{e}} / e n_{\mathrm{e}}\right)\left(\mathrm{d} n_{\mathrm{e}} / \mathrm{d} r\right)$ and $\overline{\boldsymbol{u}}_{\mathrm{e}}$ as given by $(9 a)$, describes the electron-energy losses for maintaining the ambipolar field.

The electron energy losses

$$
\frac{\delta\left(n\left\langle K_{\mathrm{e}}\right\rangle\right)}{\delta t}=-n_{\mathrm{e}} v_{*} U_{*}-n_{\mathrm{e}} v_{\mathrm{i}} U_{i}-\frac{3}{2} n_{\mathrm{e}} \delta v_{\mathrm{e}-\mathrm{n}} T_{\mathrm{e}}
$$

in (2) are through inelastic collisions for excitation (total excitation) and direct ionization, with frequencies $v_{*}$ [36] and $v_{i}$, respectively, and threshold energies $U_{*}$ (the energy for excitation of the first atom excited state) and $U_{i}$, as well as through elastic collisions (with $v_{\mathrm{e}-\mathrm{n}}$ calculated according to [42] and $\delta=2 m_{\mathrm{e}} / m_{\mathrm{a}}$ being the part of the electron energy lost in an elastic collision with atoms; $m_{\mathrm{a}}$ is the atom mass).

The final form of the electron energy balance equation is

$$
\begin{gathered}
\left(\frac{5}{2}-g_{u}\right)\left(\frac{\mu}{R}\right)^{2} D_{\mathrm{A} e} T_{\mathrm{e}}+\frac{\mu^{3}}{R^{4}} T_{\mathrm{e}} D_{\mathrm{A} e} \frac{1}{J_{1}(\mu)} \int_{0}^{R} r \frac{J_{1}^{2}(\mu r / R)}{J_{0}(\mu r / R)} \mathrm{d} r \\
+v_{*} U_{*}+v_{i} U_{i}+\frac{3}{2} \delta v_{\mathrm{e}-\mathrm{n}} T_{\mathrm{e}}=\Theta .
\end{gathered}
$$

It is obtained from equation (2) and its terms specified by (19), (20) and (22), after integration over the discharge cross section (with a boundary condition for a zero thermal flux at the discharge walls). The quantity $\Theta=\bar{Q} / \bar{n}_{\mathrm{e}}$ in the righthand side of (23) is the power absorbed on average by an electron defined through the averaged plasma density $\bar{n}_{\mathrm{e}}$ and the averaged-over the discharge cross section-Joule heating $\bar{Q}$ in the high frequency field $\left(Q=(1 / 2) \operatorname{Re} \sigma_{\tilde{\boldsymbol{E}}}|\tilde{\boldsymbol{E}}|^{2}\right.$ as given by the first term in the right-hand side of (20)). According to (23), the absorbed power $\Theta$ compensates losses due to collisions (the last three terms in the left-hand side of (23)) and fluxes of thermal energy and pressure-force work carried by the directed velocity in the ambipolar dc field (the first term in the left-hand side of (23) stemming from the second term in expression (19)) as well as losses for maintaining the ambipolar field (the second term in the left-hand side of (23) stemming from the second term in the right-hand side of (20)); $D_{\mathrm{A} e}$ is the ambipolar diffusion coefficient of the electrons as given by $(10 b)$. The power $\Theta$ is defined based on the power input to the discharge according to $(5 a)$.

\subsection{Results and discussions}

The set of equations (12)-(16) and (23) provides description of the gas-discharge part of the model giving the results for the densities of the electrons $\left(n_{\mathrm{e}}\right)$, of the atomic $\left(n_{1}\right)$ and molecular $\left(n_{2}\right)$ ions and the population $\left(N_{j}\right)$ of the excited states as well as for the power $\Theta$, the electron temperature $T_{\mathrm{e}}$ and the parameter $\mu$ of the radial plasma-density inhomogeneity. The gas-pressure range covered by the results is $p=(0.05-5)$ Torr. The power is varied up to $P=600 \mathrm{~W}$. The values of the other external parameters are $R=2.4 \mathrm{~cm}, R^{\prime}=2.6 \mathrm{~cm}, T_{\mathrm{g}}=600 \mathrm{~K}$ and $L=10 \mathrm{~cm}$.

The gas temperature $T_{\mathrm{g}}$ is an external parameter of the model, together with the gas pressure $p$. On the other hand, $T_{\mathrm{g}}$ and $p$ are related to each other via $p=\kappa T_{\mathrm{g}} N_{0}$. Since the pressure dependence of the discharge behaviour is looked for, as usually done, $p$ is the external parameter varied and the same value of $T_{\mathrm{g}}$-reasonable, according to measurements (e.g. [43]) - is taken for the total, wide, pressure range considered. In fact, the neutral atom density $N_{0}$ is the parameter basically present in the particle balance (13)-(16), not $T_{\mathrm{g}}$ and $p$. Thus, for example, reduced gas temperature is equivalent to an effective-proportional-increase in the gas pressure.

Figure 2 shows the changes of the plasma characteristics $\left(\Theta, T_{\mathrm{e}}\right.$ and electron- and ion-densities) of the discharge with varying gas pressure. With the gas-pressure increase the power $\Theta$ and the electron temperature decrease (figure $2(b)$ ), due to the decrease in the diffusion losses. Excluding the highest pressures, inelastic collisions for atom excitation are the main contributor to the electron energy losses and, respectively, to the formation of $\Theta$ over the pressure range considered. Starting with a value of more than $50 \%$ at $p=0.05$ Torr, the contribution of these losses increases close to $80 \%$ at moderate pressures ( $p=(0.2-1)$ Torr). However, with the further increase in the pressure the contribution of the losses for excitation drops to about $40 \%$ (at $p=5$ Torr). In the range of the highest gas pressure the elastic collisions gain in importance with a contribution which increases in the range $p=(1-5)$ Torr from $10 \%$ up to $50 \%$. The collisions for direct ionization provide a significant contribution, at the lowest pressure values, e.g. about $30 \%$ for $p=0.05$ Torr which is about half of the contribution of the excitation losses. The 

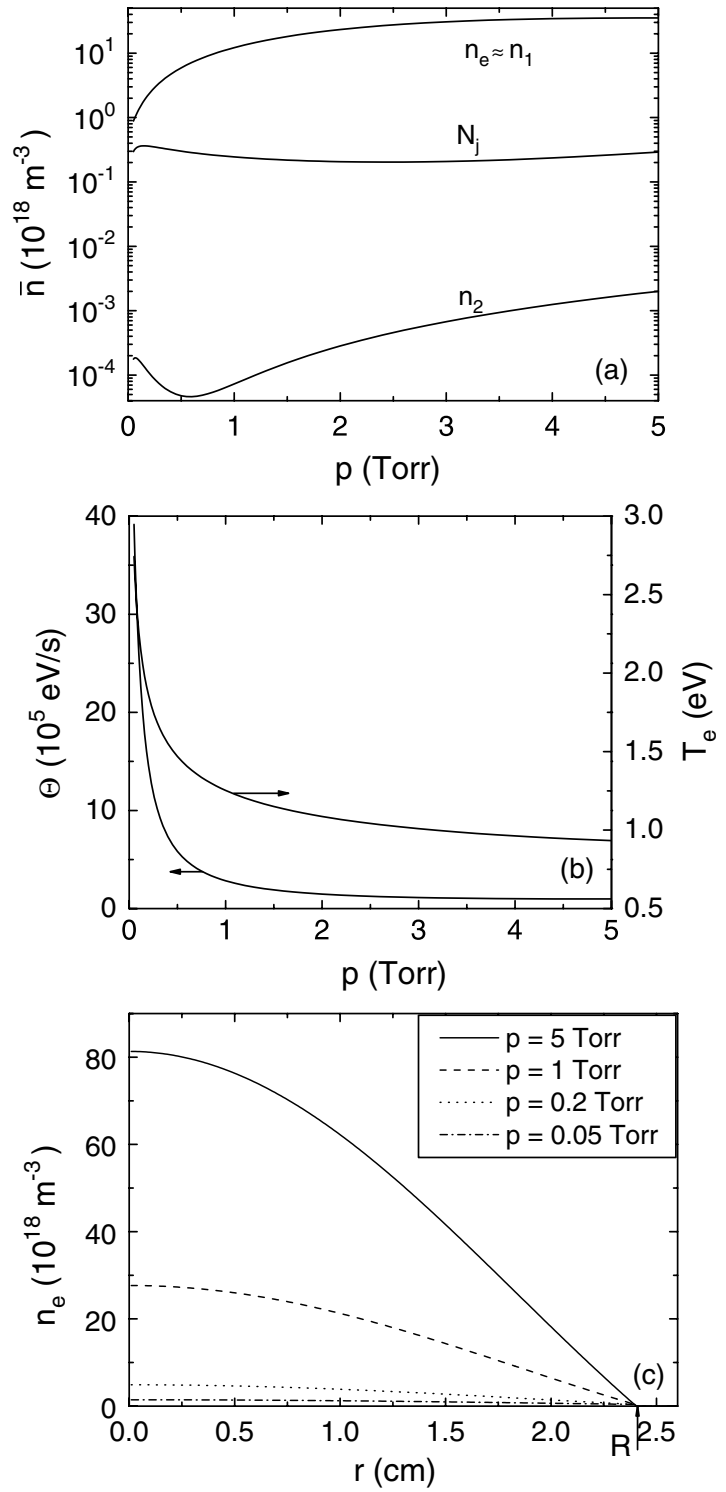

Figure 2. Variations with the gas pressure $p$ of the averaged densities of electrons $\left(n_{\mathrm{e}}\right)$, atomic $\left(n_{1}\right)$ and molecular $\left(n_{2}\right)$ ions and of the population density $N_{j}$ of the excited states in $(a)$ and of $\Theta$ and $T_{\mathrm{e}}$ in $(b) ;(c)$ radial profiles of the plasma density for different pressure values. Constant applied power $P=100 \mathrm{~W}$. The position of the internal radius of the gas-discharge tube is marked in $(c)$.

rest is provided by losses due to fluxes of thermal energy and pressure-force work carried by the directed velocity in the ambipolar field. Having the highest value of about $10 \%$ for $p=0.05$ Torr, the contribution of these losses decreases with the gas-pressure increase. The contribution of the losses for maintaining the ambipolar field stays at a value of about $7 \%$ up to $p=2$ Torr and then decreases with the $p$-increase.

With the constant value of the applied power, the $\Theta$ decrease leads to an increase in the plasma density $n_{\mathrm{e}}$ (figure 2(a)). The averaged concentrations of electrons and atomic ions are almost the same. Due to the high plasma density, the step ionization is the major contributor to the production of electrons and atomic ions for $p \geqslant 0.5$ Torr. The contribution of the direct ionization is higher than that of the step ionization for $p \leqslant 0.2$ Torr. The losses of electrons and

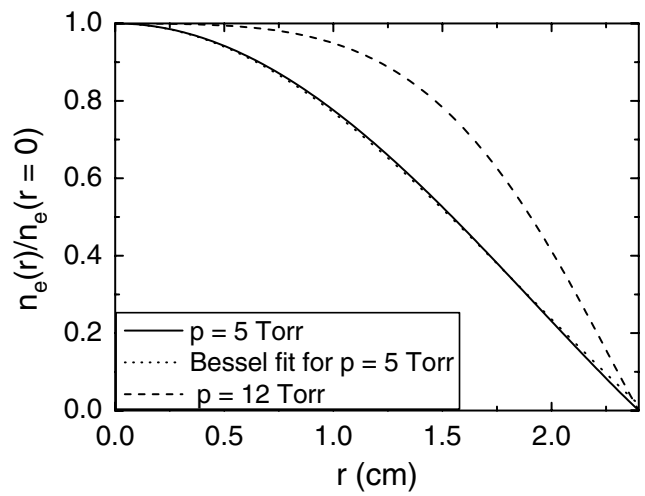

Figure 3. Normalized radial profiles of the electron concentration obtained by solving the balance equations of the charged particles and of the electron energy in their form (1) and (3) of differential equations, together with the Poisson equation. Gas-pressure values: $p=5$ Torr (full curve) and 12 Torr (broken curve). The dotted curve shows the Bessel-function approximation to the profile at $p=5$ Torr.

atomic ions are mainly through ambipolar diffusion confirming that the pressure range chosen is governed by a diffusioncontrolled regime. At the highest pressures, close to $p=$ 5 Torr, a transition to a recombination-controlled regime shows up: the contribution of the DR becomes also important in the electron balance reaching half of the contribution of the diffusion. In this pressure range the AAA provides a significant contribution to the losses of the atomic ions.

Even with recombination losses reaching-for $p=$ 5 Torr - half of the ambipolar diffusion losses, the radial profile of the plasma density is still a Bessel-type of profile, as figure 3 shows. The results in figure 3 are direct solutions of the differential equations (1) and (2), combined in a set with the Poisson equation. Obtaining a Bessel-type of profile when the diffusion is the main mechanism of losses proves the validity of the simplifications discussed in subsection 3.1.3, checked also before [19], justifying the applicability of the model discussed here. However, with the further increase in the gas-pressure-above the gas-pressure range of the diffusioncontrolled discharges considered here-the growing role of the recombination shows up in flattening of the radial profile of the plasma density (figure 3 ) in the central part of the discharge.

The variation with $p$ of the concentration $n_{2}$ of the molecular ions is nonmonotonic (figure $2(a)$ ). This is due to a redistribution of the processes responsible for the $\mathrm{Ar}_{2}^{+}$-balance. In the lower pressure range the losses of $\mathrm{Ar}_{2}^{+}$-ions, mainly due to dissociation by electron impact (DEI), are compensated by MAI. In the higher pressure range the losses are by both DEI and DR and they are compensated by AAA. The transition between production by MAI to production by AAA is at about $p=0.5$ Torr as it could be seen also in figure 2(a). The contribution of dissociation by atom impact (DAI) is negligible.

Up to $p \cong 2$ Torr the density of the excited states (figure $2(a)$ ) is determined by population from the ground state and depopulation by step ionization. However, for higher pressures a contribution of the DR shows up in their production. Although the atomic model employed here is very simplified, the values of the population of the metastable states are of the 

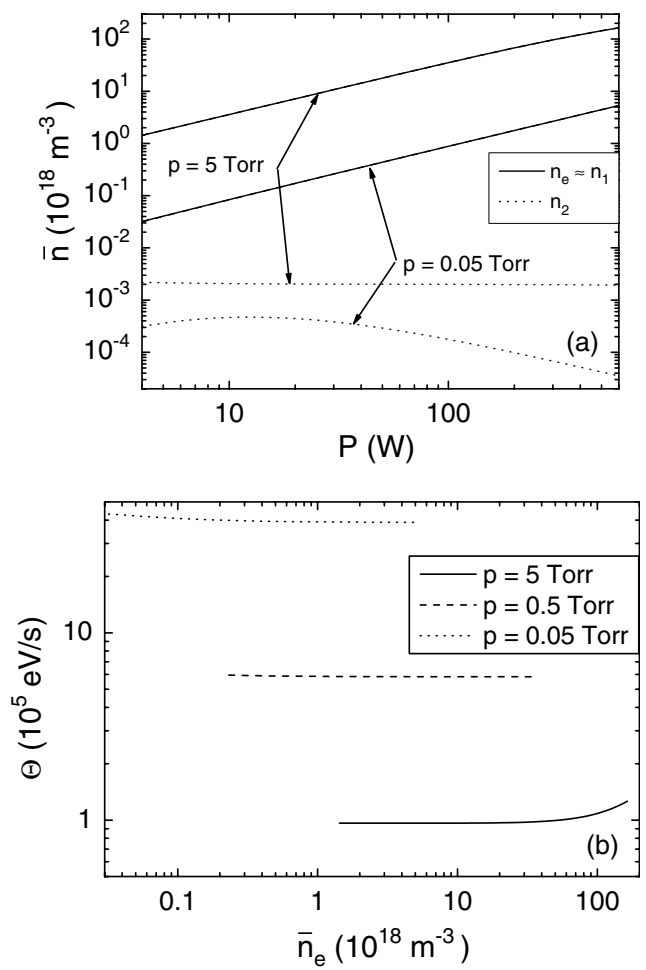

Figure 4. Variations with the applied power $P$ of the averaged densities of electrons $\left(n_{\mathrm{e}}\right)$, atomic $\left(n_{1}\right)$ and molecular $\left(n_{2}\right)$ ions in (a) and of $\Theta$, presented in terms of $\bar{n}_{\mathrm{e}}$-variation, in $(b)$. Constant pressure values: $p=0.05$ and 5 Torr in $(a)$ and $p=0.05,0.5$ and 5 Torr in $(b)$.

same order of magnitude compared with results from quite more detailed collisional-radiative models (e.g. [44]). This may be attributed to partial compensation of the contributions of cascades from higher levels and losses to the resonance states which, beyond all question being important, are mechanisms acting in the opposite directions. In fact, the differences in the results for the population of the metastable states obtained from the different models show evidence mainly at low gas pressure, a case when, as it has been mentioned before, the contribution to the charged particle balance of the direct ionization becomes bigger than that of the step ionization.

The obtained values (figure 2) of the plasma density are of the order of those given in [8] for the same value $(P=100 \mathrm{~W})$ of the applied power. Since the plasma-density production in the inductive discharges is high, the conditions of their maintenance are far from validity of the Schottky condition, thus requiring modelling, as it is done here, involving the concept [19-26] of generalization of the Schottky condition which calls for the importance of the nonlinear processes of step ionization and recombination and, in the case of the argon discharges considered here, of the nonlinear processes accompanying the DR.

Figure 4 shows changes in the behaviour of the plasma characteristics with variation of the applied power $P$. The plasma density increases with the applied power (figure $3(a)$ ). Due to the slight dependence of $\Theta$ on $\bar{n}_{\mathrm{e}}$ (figure $4(b)$ ), respectively, on $P$, this increase is almost proportional to the increase of $P$ (figure 4(a)). The obtained results for the plasma density for the conditions of the experiment in

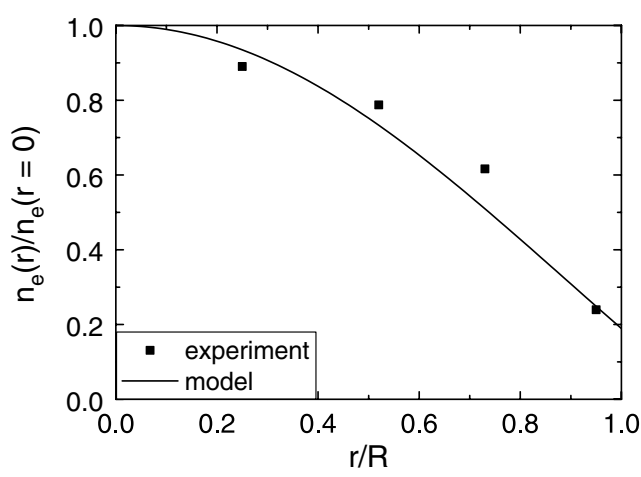

Figure 5. Normalized radial profile of the electron concentration for $p=100$ mTorr, $P=350 \mathrm{~W}$ and $R=1.9 \mathrm{~cm}$ : theoretical result from the model presented here (full curve) and experimental data from [45] (symbols).

[45] are of the same order of magnitude, with a difference less than a factor of 2, compared with the data measured. The comparison (figure 5) of the type of the radial profile obtained experimentally and theoretically also shows very good agreement. Estimations of the power $\Theta$ from data for the plasma density from experiments in inductive discharges with planar coils $[46,47]$, made by involving the values of the measured applied power and the size of the discharge vessel, are also in agreement with the obtained theoretical values. The constant value of $\Theta$ at moderate pressure (figure $4(b)$ ) is due to the saturation in the step ionization. The $\Theta$-increase with decreasing density when the gas pressure is low is due to step ionization acting as a nonlinear process, i.e. without saturation. The $\Theta$-increase with the $\bar{n}$-increase when the gas pressure is high is due to increasing contribution of the recombination losses.

The results show that, due to the high densities in the inductive discharge, the step ionization is saturated, i.e. it appears as an almost linear process not providing a strong evidence of a $(\Theta-n)$-dependence. However, going beyond the Schottky condition and accounting for step ionization and also for DR (and the processes involved by it) in the modelling of argon discharges is a requisite because these are the processes which, together with the diffusion losses, govern the discharge behaviour. This shows up both in the particle balance and in the values of $\Theta$ which are from 2 (for $p=0.05$ Torr) up to 5 (for $p=5$ Torr) times lower than those predicted by the Schottky condition.

\section{Electrodynamics and power deposition into the discharge}

The electrodynamical part of the model completes its gas discharge part (section 3) with the radial distribution of the components $\left(E_{\varphi}\right.$ and $\left.H_{z}\right)$ of the high-frequency field sustaining the discharge. Moreover, the plasma density and the field distribution obtained, respectively, in the gas-discharge description and in the electrodynamical description of the discharge provide the results for the spatial distribution of the high-frequency current density $j_{\varphi}$ and of the power input (i.e. the Joule heating) into the discharge. Regarding the discussions on the maintenance of the inductive discharges, the skin depth, also given here, is the other important quantity. 
The results for the changes of the parameters of the external coil due to the plasma loading in the coil are also presented.

\subsection{Set of equations}

The space distribution of the field sustaining the discharge stems from the wave equation (4) written-in cylindrical coordinates-for the $H_{z}$-field component of the transverse highfrequency wave producing the inductive discharge:

$$
\begin{gathered}
\frac{\mathrm{d}^{2} H_{z}(r)}{\mathrm{d} r^{2}}+\frac{1}{r} \frac{\mathrm{d} H_{z}(r)}{\mathrm{d} r}-\frac{1}{\varepsilon(r)} \frac{\mathrm{d} \varepsilon(r)}{\mathrm{d} r} \frac{\mathrm{d} H_{z}(r)}{\mathrm{d} r} \\
+\frac{\omega^{2}}{c^{2}} \varepsilon(r) H_{z}(r)=0 .
\end{gathered}
$$

The radial plasma-density inhomogeneity of the diffusioncontrolled discharges is taken into account in (24). The highfrequency electric field of the wave can be obtained from the relation

$$
E_{\varphi}(r)=-\frac{\mathrm{i}}{\omega \varepsilon_{0} \varepsilon(r)} \frac{\mathrm{d} H_{z}(r)}{\mathrm{d} r}
$$

between the two field components; $\varepsilon_{0}$ is the vacuum permittivity. According to expression (21) for the conductivity, the plasma permittivity is

$$
\varepsilon(r)=1-\frac{\mathrm{i} \omega_{\mathrm{p}}^{2}(r)}{\omega} \frac{4 \pi}{3} \int_{0}^{\infty} \frac{v^{3}}{-\mathrm{i} \omega+v_{\mathrm{e}-\mathrm{n}}} \frac{\mathrm{d} f_{0}}{\mathrm{~d} v} \mathrm{~d} v,
$$

where the plasma frequency $\omega_{\mathrm{p}}$ involves the electron density (11) obtained in the gas-discharge part of the model. The velocity-dependence of $v_{\mathrm{e}-\mathrm{n}}$ taken into account in the plasma conductivity (21) crosses into the plasma permittivity, influencing the electrodynamical description of the discharge.

Equation (24) is numerically solved in the plasma volume with boundary conditions at the discharge axis:

$$
\begin{aligned}
& \mathrm{d} H_{z} /\left.\mathrm{d} r\right|_{r=0}=0, \\
& H_{z}(r=0)=H_{0},
\end{aligned}
$$

where $H_{0}$ is the value of the magnetic field which provides an electric field satisfying the power conservation $(5 b)$.

The analytical solutions for the field distribution in the glass tube $\left(R \leqslant r \leqslant R^{\prime}\right)$ are

$$
\begin{aligned}
& H_{z}\left(R \leqslant r \leqslant R^{\prime}\right)=C_{1} J_{0}\left(\frac{\omega}{c} \sqrt{\varepsilon_{\mathrm{g}}} r\right)+C_{2} Y_{0}\left(\frac{\omega}{c} \sqrt{\varepsilon_{\mathrm{g}}} r\right), \\
& E_{\varphi}\left(R \leqslant r \leqslant R^{\prime}\right)=\frac{\mathrm{i}}{c \varepsilon_{0} \sqrt{\varepsilon_{\mathrm{g}}}}\left[C_{1} J_{1}\left(\frac{\omega}{c} \sqrt{\varepsilon_{\mathrm{g}}} r\right)\right. \\
& \left.\quad+C_{2} Y_{1}\left(\frac{\omega}{c} \sqrt{\varepsilon_{\mathrm{g}}} r\right)\right],
\end{aligned}
$$

where $\varepsilon_{\mathrm{g}}$ is the permittivity of the tube $\left(\varepsilon_{\mathrm{g}}=3.8\right)$. The constants $C_{1}$ and $C_{2}$ are determined by matching - at $r=R-$ these solutions to the solutions in the plasma region.

The skin depth of the transverse high-frequency wave which produces the over-dense plasmas $\left(n_{\mathrm{e}}>n_{\mathrm{cr}}\right.$ where $n_{\mathrm{cr}}$ is the critical density) of the inductive discharges is calculated from the radial distribution of the $E_{\varphi}$-field according to

$$
\delta_{\mathrm{s}}=\frac{1}{E_{\varphi}(r=R)} \int_{0}^{R} E_{\varphi}(r) \mathrm{d} r .
$$

\subsection{Results and discussions}

The results from the electrodynamical part of the model presented here are for the radial distribution of the electromagnetic field components, as well as for the wave power deposition, i.e. for the Joule heating, and for the current density in the discharge. Results for the plasma conductivity, for the skin depth and for the total current in the discharge are also discussed. The importance of accounting for the plasma density inhomogeneity is also stressed. The analysis of the results is in terms of varying gas pressure $p$, applied power $P$ and frequency $\omega$. The results for different $p$ - and $P$-values complete the results for the plasma characteristics of the discharge in figures 2 and 4 to a self-consistent model of a $13.56 \mathrm{MHz}$ discharge. In the gas pressure range considered the discharge maintenance is under the conditions of strong collisions (electron-neutral elastic collision frequency larger than the wave frequency). Since changes of the frequency of the field maintaining the discharge influence only the electrodynamical part of the model, the results for different $\omega$ values combined with the results for the plasma characteristics for given $p$ and $P$ (section 3 ) show the changes of the discharge structure with varying frequency of the high-frequency field.

4.2.1. Varying gas pressure. The field distribution in figure 6 is in discharges sustained at $f=\omega / 2 \pi=13.56 \mathrm{MHz}$, $P=100 \mathrm{~W}$ and different gas pressures $p$ (figure 2). Figure $6(a)$ shows the radial decay into the plasma region of the field components. The changes of the amplitude of the field components with the increase in the gas-pressure are nonmonotonic: with the $p$-increase from 0.05 till 2 Torr the field amplitudes decrease and then, above 2 Torr, they slightly increase. This is due to the nonmonotonic changes of the real part of the plasma conductivity (figure $6(b)$ ). For discharge maintenance under the conditions of strong collisions, the dependence of $\operatorname{Re}\left(\sigma_{\tilde{\boldsymbol{E}}}\right)$ on the plasma density $n_{\mathrm{e}}$ and on the elastic electron-neutral collision frequency is of the type of $\operatorname{Re}\left(\sigma_{\tilde{\boldsymbol{E}}}\right) \propto n_{\mathrm{e}} / \nu_{\mathrm{e}-\mathrm{n}}(21)$. Both $\nu_{\mathrm{e}-\mathrm{n}}$ and $n_{\mathrm{e}}$ (figure $2(a)$ ) increase with $p$. However, $n_{\mathrm{e}}$ increases strongly in the lower pressure range, and slightly in the higher pressure range. This determines the appearance of a maximum in the dependence of $\operatorname{Re}\left(\sigma_{\tilde{E}}\right)$ on $p$ (figure $6(b)$ ). In a combination with the constant value of the power applied for the discharge maintenance $P=(1 / 2) \int_{(\mathrm{V})} \operatorname{Re}\left(\sigma_{\tilde{E}}\right)\left|E_{\varphi}\right|^{2} \mathrm{~d} V=$ const. (relation $(5 b)$ ), this behaviour of $\operatorname{Re}\left(\sigma_{\tilde{E}}\right)$ should result in the nonmonotonic changes of the amplitude of the $E_{\varphi}$-field shown in figure $6(a)$. The relation of the $H_{z}$-field to the $E_{\varphi}$-field (25) explains the corresponding nonmonotonic changes of the amplitude of the $H_{z}$-field. The nonmonotonic variation of the skin depth $\delta_{\mathrm{s}}$ with increasing gas pressure (figure $6(b)$ ) correlates with the behaviour of $\operatorname{Re}\left(\sigma_{\tilde{E}}\right)$. It is also due to the combined increase in $n_{\mathrm{e}}$ and $v_{\mathrm{e}-\mathrm{n}}$ with $p$. When the collisions are strong, the skin length $\delta_{\mathrm{s}}$ is

$$
\delta_{\mathrm{s}}=\left(2 \tilde{v}_{\mathrm{e}-\mathrm{n}} / \tilde{\omega}\right)^{1 / 2}\left(c / \omega_{\mathrm{p}}\right),
$$

where $\tilde{\nu}_{\mathrm{e}-\mathrm{n}}$ and $\tilde{\omega}$ are, respectively, effective elastic electronneutral collision frequency and wave frequency $[27,28]$. The dependence $\delta_{s} \propto\left(\tilde{v}_{\mathrm{e}-\mathrm{n}} / n_{\mathrm{e}}\right)^{1 / 2}$ determines a decrease in $\delta_{\mathrm{s}}$ in the lower pressure range and its increase with $p$ in the highpressure range. 

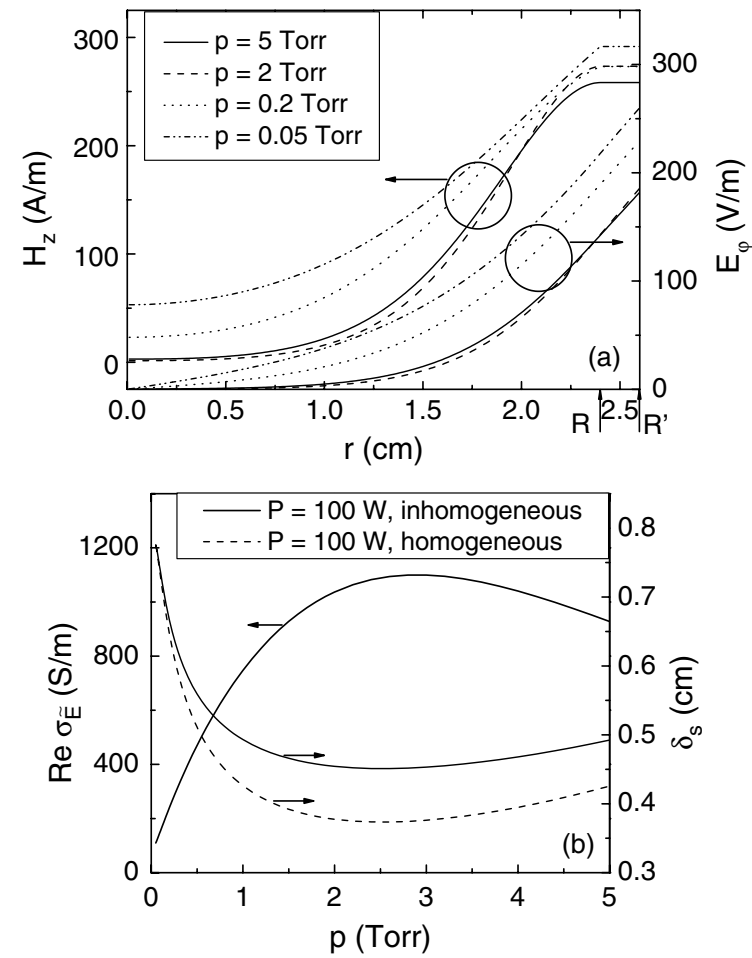

Figure 6. (a) Radial profiles of the amplitudes of the $E_{\varphi}$-field and $H_{z}$-field components for different values $(p=0.05,0.2,2$ and 5 Torr) of the gas pressure. (b) Variation with the gas pressure of the real part of the plasma conductivity $\left(\operatorname{Re} \sigma_{\tilde{E}}\right)$ and of the skin depth calculated for inhomogeneous plasma (full curve) and homogeneous plasma (broken curve) with plasma density equal to the averaged-over the discharge cross section-density of the inhomogeneous plasma. Frequency $f=13.56 \mathrm{MHz}$ of the field maintaining the discharge. Discharges with plasma characteristics presented in figure 2: $P=100 \mathrm{~W}$ and the corresponding $p$-values. The position of the internal and external radii of the gas-discharge tube is marked in $(a)$.

The Joule heating $Q(r)=(1 / 2)\left(\operatorname{Re} \sigma_{\tilde{E}}\right)\left|E_{\varphi}(r)\right|^{2}$ is localized close to the walls (figure $7(a)$ ) as it should be expected for inductive discharges. The same concerns the current density $j_{\varphi}=\sigma_{\tilde{E}} E_{\varphi}$ (figure $7(b)$ ). According to the dependence of $Q$ and $j_{\varphi}$ on $E_{\varphi}$, the Joule heating is stronger concentrated in the outer region of the discharge than the current density. The appearance of the maximum of both $Q\left(Q(r) \propto n_{\mathrm{e}}\left|E_{\varphi}\right|^{2}\right)$ and $j_{\varphi}$ in the outer region of the discharge is due to the simultaneous decrease in the plasma density (figure 2(c)) and increase in the electric field (figure 6(a)) over the radius.

With increasing gas pressure the maxima of $Q$ and $j_{\varphi}$ shift towards the discharge axis (figures $7(a)$ and $(b)$ ). The irregular distribution of the peak values of $Q$ (and $j_{\varphi}$ ) for different gas pressures may be related to the nonmonotonic variation with $p$ of the real part of the plasma conductivity (figure $6(b)$ ). The total current $I_{\mathrm{pl}}=L \int_{0}^{R} j_{\varphi} \mathrm{d} r$ decreases with increasing $p$ (figure $7(c)$ ) as it could be also estimated from $I_{\mathrm{pl}}=L j_{\varphi} \delta_{\mathrm{s}}$ (with $j_{\varphi}$ and $\delta_{\mathrm{s}}$ shown, respectively, in figures $7(b)$ and $6(b)$ ).

4.2.2. Varying applied power. The results in figure 8 combined with those in figure 4 show the changes of the structure of a $13.56 \mathrm{MHz}$ discharge with the variation of the power $P$ applied for the discharge maintenance. The
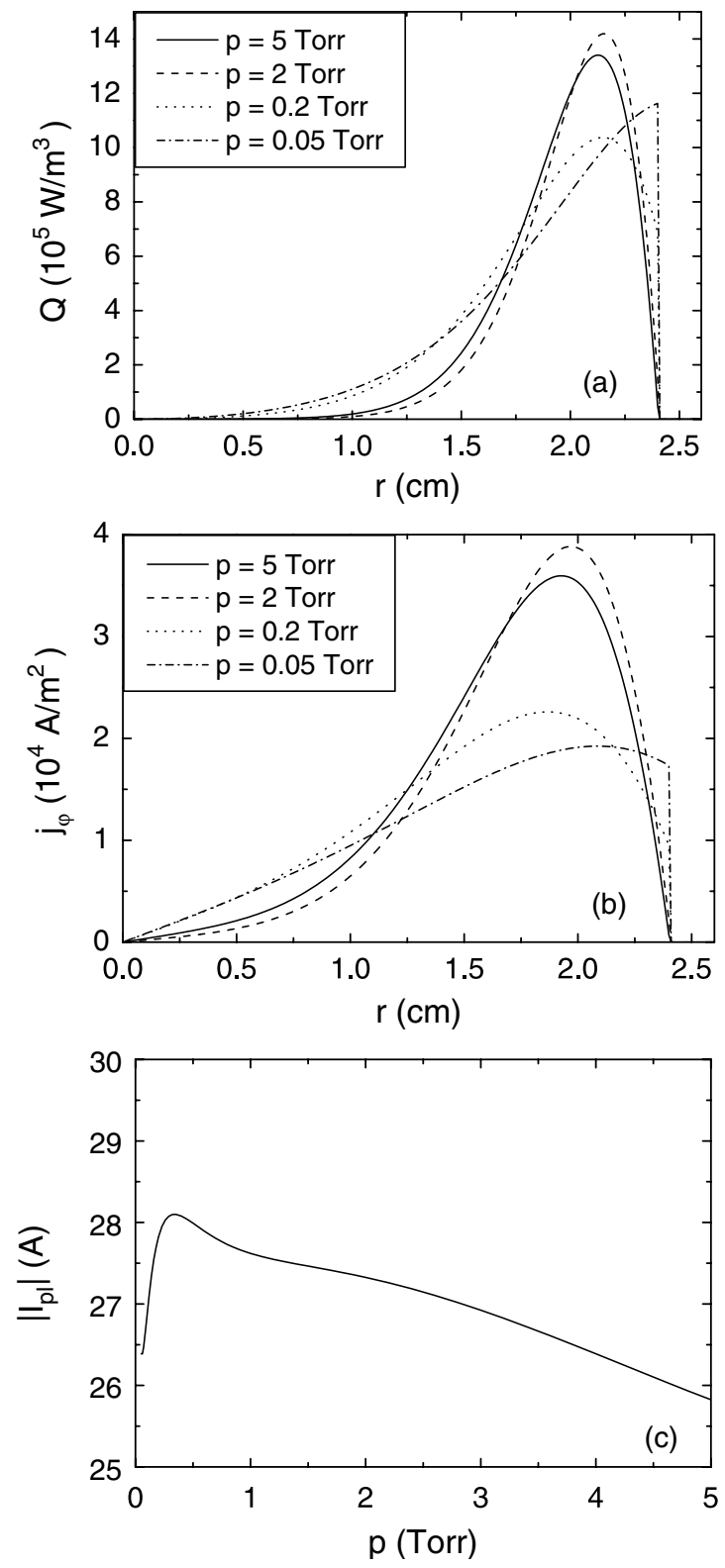

Figure 7. Radial distribution of the Joule heating $Q$ in $(a)$ and of the current density $j_{\varphi}$ in $(b)$ for different $p$-values, as indicated in the figures, of the discharges in figures 2 and $6(P=100 \mathrm{~W}$, $f=13.56 \mathrm{MHz}$ ). (c) Total current in the discharge.

amplitudes of the field components in the out-off centre region monotonically increase with the $P$-increase (figure $8(a)$ ). Due to the density increase with $P$, the skin depth decreases (figure 8(b)).

Figure 9 shows the expected increase in the peak value of $Q$ with increasing $P$ (according to expressions $(5 a)$ and (5b) for the power conservation, $\left.P=2 \pi L \int_{0}^{R} r Q(r) \mathrm{d} r\right)$. Respectively, the peak value of $j_{\varphi}$ also increases with $P$. Decreasing applied power causes a slight shift of the peak values of $Q$ and $j_{\varphi}$ towards the discharge axis. The variation of the total current in the plasma with $P$ is given in figure $8(b)$.

4.2.3. Varying wave frequency. Figures 10 and 11 show results of the discharge operation for different values of the 

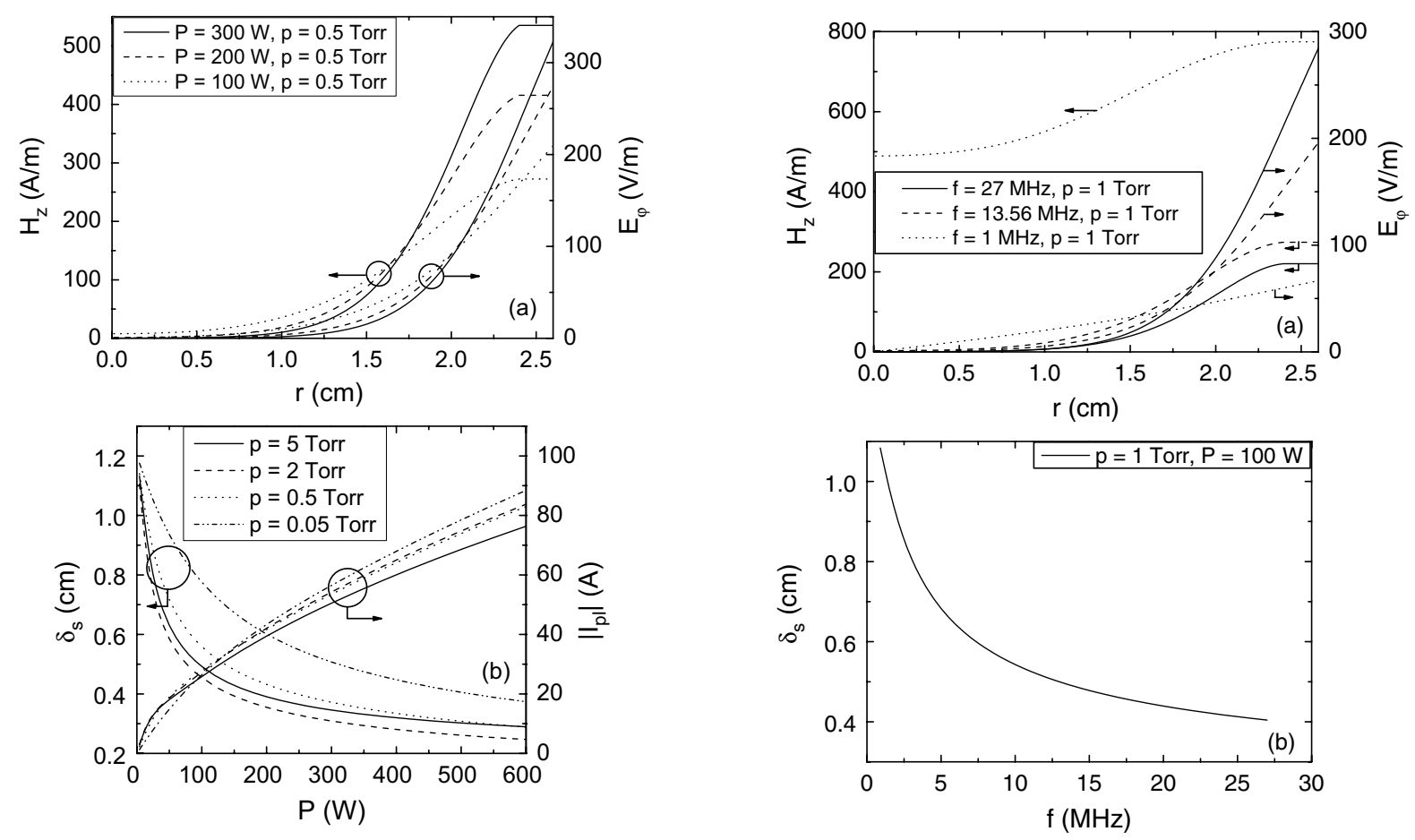

Figure 8. (a) Radial profiles of the amplitudes of the $E_{\varphi}$-field and $H_{z}$-field components for different values $(P=100,200$ and $300 \mathrm{~W}$ ) of the applied power. $(b)$ Variation with the applied power of the skin depth $\delta_{\mathrm{s}}$ and of the total current $I_{\mathrm{pl}}$ in the discharge. Frequency $f=13.56 \mathrm{MHz}$ of the field maintaining the discharge. Discharges with plasma characteristics presented in figure 4 for the corresponding values of the gas pressure.

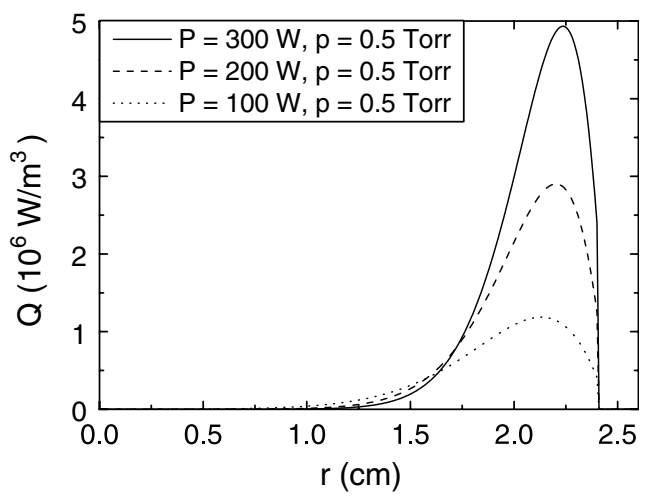

Figure 9. Radial distribution of the Joule heating for different $P$-values of the discharges in figures 4 and 8 (for $p=0.5$ Torr, $f=13.56 \mathrm{MHz})$.

frequency $f$ of the driving current in the coil. The skin depth $\delta_{\mathrm{s}}$ decreases with increasing $f$ (figure $10(b)$ ). The results obtained from (29) are well approximated by the $\left(\tilde{\omega}^{-1 / 2}\right)$ dependence of $\delta_{\mathrm{s}}$ predicted by (30). Due to the strong collisions, the real part of the plasma conductivity (21) does not depend on $\omega$. Therefore, for the constant value of the applied power considered, and with the decrease of $\delta_{\mathrm{s}}$ with increasing $f$, the amplitude of the $E_{\varphi}$-field should increase with $f$, as figure $10(a)$ shows. The increase in $E_{\varphi}$ leadsaccording to (25) — to the increase in the slope of the $H_{z}$-profile (figure $10(a)$ ). Since a magnetic field with lower frequency induces an electric field with lower magnitude, the amplitude

Figure 10. (a) Radial profiles of the amplitudes of the $E_{\varphi}$-field and $H_{z}$-field components for different values of the frequency ( $f=1$, 13.56 and $27 \mathrm{MHz}$ ) of the field maintaining the discharge. $(b)$ Variation with the frequency of the skin depth. Discharges with plasma characteristics presented in figure 2 for $P=100 \mathrm{~W}$ and the corresponding $p$-value ( $p=1$ Torr).

of the magnetic field should be higher at low frequencies (as figure $10(a)$ shows) in order to keep the same value of the power $P$.

The strong penetration of the electric field into the plasma (figure 10) when the frequency is low leads to a shift of both $Q$ and $j_{\varphi}$ (figure 11) towards the central part of the discharge.

4.2.4. Influence of plasma-density inhomogeneity. The necessity of accounting for the inhomogeneity of the plasma density in the electrodynamical part of models of diffusioncontrolled discharges is illustrated in figures 12 and 13. The 'homogeneous' case is calculated by using a radially constant plasma density with a value equal to the averaged plasma density obtained from the Bessel function radial profile (11). In the inductively coupled discharges most of the power of the electric field is deposited in the outer region of the plasma column, i.e. close to the walls. Therefore, it can be expected that replacing the plasma density drop close to the wall of the Bessel-type of profile (figure 2(c)) by the quite higher value of the averaged density may cause significant changes. This is confirmed by the results for the radial profiles of the electric (figure 12(a)) and magnetic (figure 12(b)) fields. With the constant value of the applied power kept, the artificial increase in the plasma density and, respectively, of the real part of the plasma conductivity (21) when an averaged density is used leads to the lower values of $E_{\varphi}$ shown in figure $12(a)$ in the 'homogeneous' case. This means that the magnetic field which induces the electric field should be also smaller, in the case of homogeneous plasmas, as figure 12(b) shows. Replacing the plasma density drop in the outer region of the discharge with 

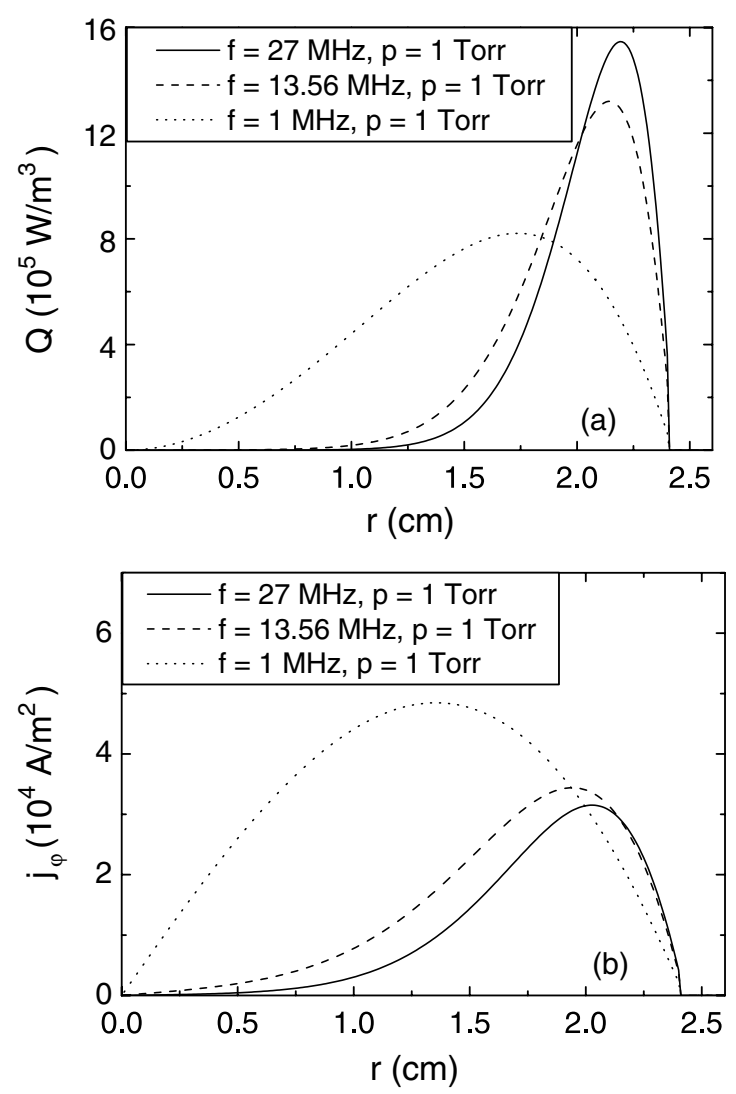

Figure 11. Radial distribution of the Joule heating $Q$ in $(a)$ and of the current density $j_{\varphi}$ in $(b)$ for different $f$-values, as indicated in the figures, of the discharges in figures 2 and 10 for $p=1$ Torr, $P=100 \mathrm{~W}$.

the averaged density leads to a smaller skin depth (figure $6(b)$ ) which is in accordance also with the $\left(\delta_{\mathrm{s}} \propto n^{-1 / 2}\right)$-dependence.

Figure 13, where results for the radial distribution of $Q$ and $j_{\varphi}$ are compared for radially inhomogeneous and homogeneous plasmas, confirms that the peaks of $Q$ and $j_{\varphi}$ in inhomogeneous plasmas are due to the combined behaviour of decreasing $n_{\mathrm{e}}(r)$ and increasing $E_{\varphi}(r)$ towards the discharge walls.

\subsection{Changes of the parameters of the external coil}

As it is known $[1,8,15,16]$, the loading of plasma in the coil changes its parameters, causing a decrease (with $\Delta L$ ) in the inductance of the coil ( $N$-turn coil) and an increase in its resistance $R_{0}$ (with $\Delta R$ ), as indicated in figure 14 which shows the transformer representation of the inductive discharge and its equivalent circuit. The changes of the discharge impedance, i.e. $\Delta L$ and $\Delta R$, are the quantities needed for the estimation of all other characteristics of the circuit (current, voltage and phase difference between them). The results presented here are on the variation of $\Delta L$ and $\Delta R$ with the applied power and gas pressure. They are obtained by using the electrodynamical part of the model.

With the value of the magnetic field at the outer radius of the quartz tube known from the electrodynamical part of the model, the current in the coil, which creates this magnetic field, can be calculated according to $I_{1}=H_{z}\left(R^{\prime}\right) L / N$. The total power $P$ absorbed in the plasma is given. Therefore, the
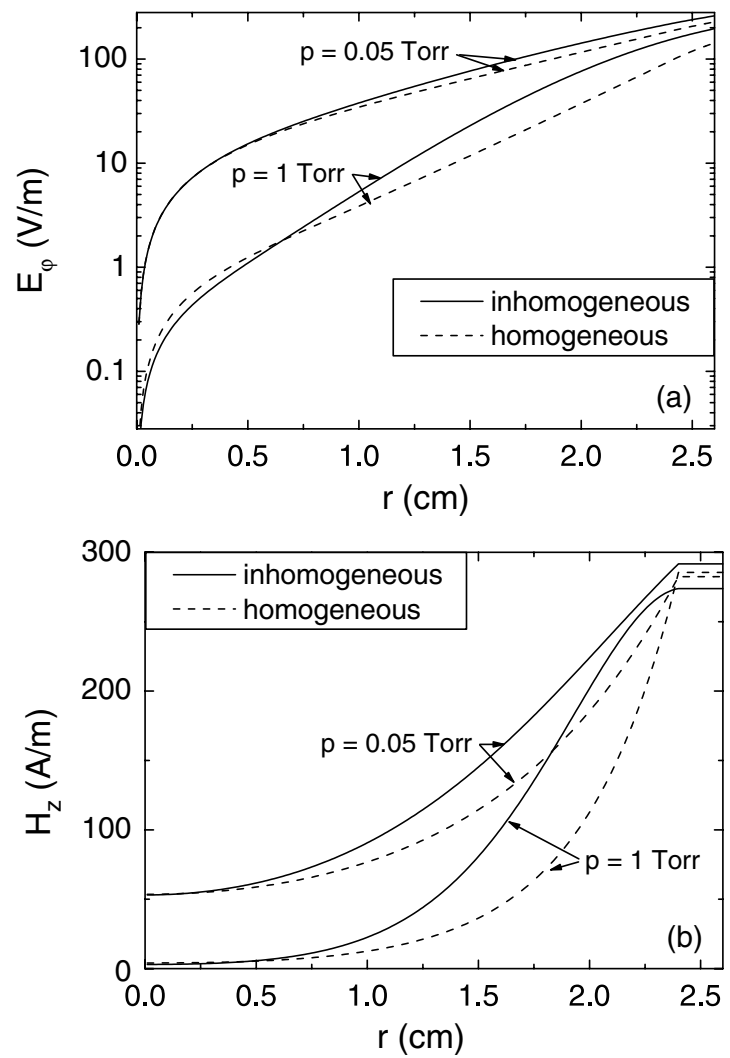

Figure 12. Radial profiles of the amplitudes of the $E_{\varphi}$-(in $\left.(a)\right)$ and $H_{z}$-(in $(b)$ ) field components calculated for inhomogeneous plasma (full curves) and homogeneous plasma (broken curves). The latter is with plasma density equal to the averaged-over the discharge cross section-density of the inhomogeneous plasma. Frequency $f=13.56 \mathrm{MHz}$ of the field maintaining the discharge. Discharges with plasma characteristics presented in figure $2: P=100 \mathrm{~W}$ and the corresponding values of $p$.

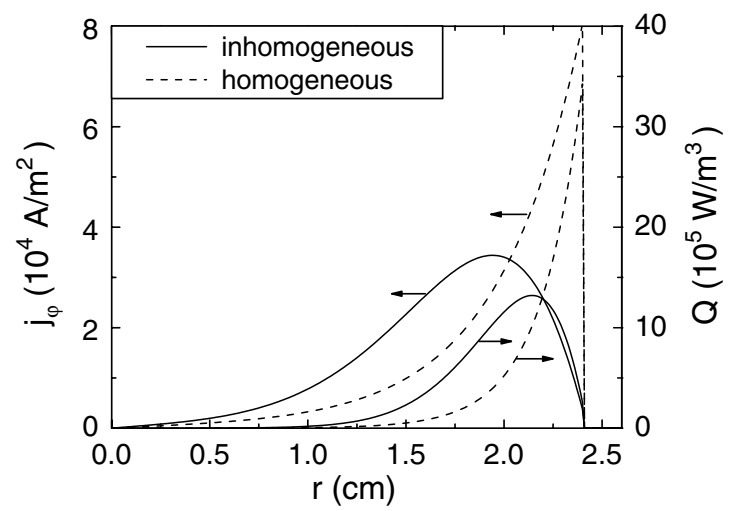

Figure 13. Comparison of results for the radial distribution of the Joule heating $Q$ and of the current density $j_{\varphi}$ in radially inhomogeneous plasma (full curves) and homogeneous plasma (broken curves). The latter is with plasma density equal to the averaged - over the discharge cross section-density of the inhomogeneous plasma. Discharge with plasma characteristics presented in figures 2 and 12.

resistance which is incorporated in the circuit due to the plasma loading in the coil is $\Delta R=2 P / I_{1}^{2}$. In order to calculate $\Delta L, L_{1}$ which is connected to the total reactive power $Q_{i}$ in the discharge $\left(Q_{i}=(1 / 2) \omega L_{1} I_{1}^{2}\right)$ should be found. Since the 


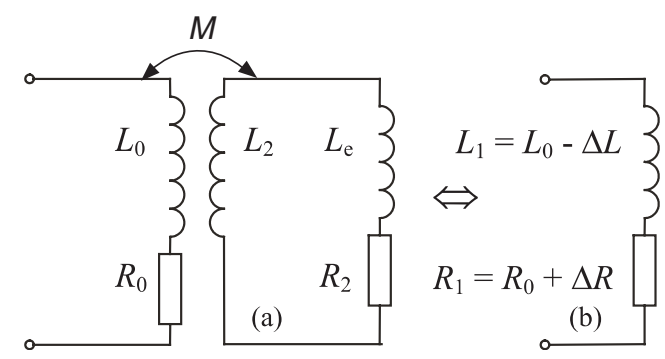

Figure 14. The transformer circuit representation of the inductive discharge in $(a)$ and its equivalent circuit in $(b)$. The second circuit in (a) presents the plasma column: $L_{2}$ and $L_{\mathrm{e}}$ are, respectively, the inductances due to the circular flow of the azimuthal currents in the plasma and to the inertia of the electrons; $R_{2}$ is the active resistance of the plasma. $M$ is the mutual inductance. In $(b), \Delta L$ and $\Delta R$ are the changes in the primary circuit impedance due to the plasma loading.

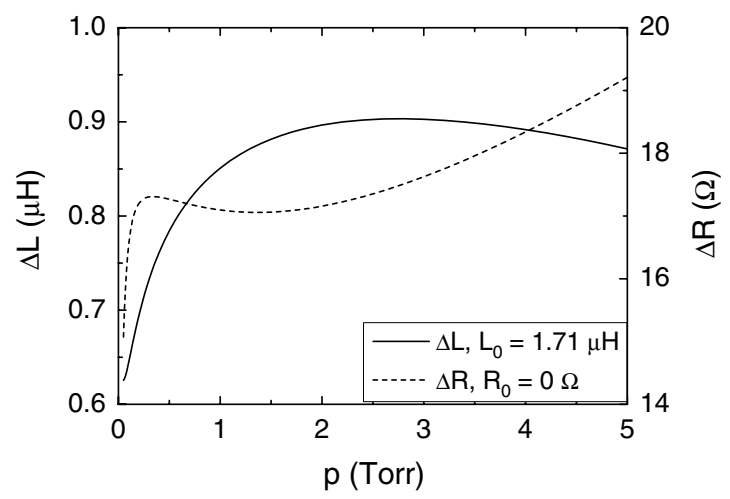

Figure 15. Variation with the gas pressure $p$ of the changes $\Delta L$ and $\Delta R$ of the inductance and the resistance of the primary circuit due to the presence of plasma.

electrodynamical part of the model provides the results for the magnetic and electric fields, the power flux in the discharge (the Poynting vector) can be easily obtained. The reactive power $Q_{i}$ which goes into the discharge is the imaginary part of the Poynting vector integrated over the surrounding surface:

$Q_{i}=\frac{1}{2} \operatorname{Im} \int_{S}\left(E \times H^{*}\right) \mathrm{d} S=\pi R^{\prime} L \operatorname{Im}\left[E_{\varphi}\left(R^{\prime}\right) H_{z}^{*}\left(R^{\prime}\right)\right]$.

Thus, $L_{1}$ is

$$
L_{1}=\frac{2 \pi}{\omega I_{1}^{2}} R^{\prime} L \operatorname{Im}\left[E_{\varphi}\left(R^{\prime}\right) H_{z}^{*}\left(R^{\prime}\right)\right]
$$

and the change $\Delta L=L_{0}-L_{1}$ of the inductance of the coil due to the plasma loading in the coil can be obtained.

The inductance of the coil (with $N=8, R^{\prime}=2.6 \mathrm{~cm}$ and $L=10 \mathrm{~cm}$ ) is $L_{0}=1.71 \mu \mathrm{H}$. Figure 15 shows the results obtained for $\Delta L$ at $P=100 \mathrm{~W}$ and varying gas-pressure. $\Delta L$ increases with the increase in the gas pressure in the pressure range from 0.05 to 2 Torr and decreases at higher pressures (from 3 to 5 Torr). This is due to the behaviour of the skin depth (figure $6(b)$ ) determined by the nonmonotonic changes of $\operatorname{Re}\left(\sigma_{\tilde{\boldsymbol{E}}}\right)$, as has already been commented. The skin depth determines approximately the radius of the oneturn coil, which is actually the plasma. Therefore, at higher skin depth the inductance of the plasma is smaller (smaller radius), resulting in smaller $\Delta L$ and vice versa.
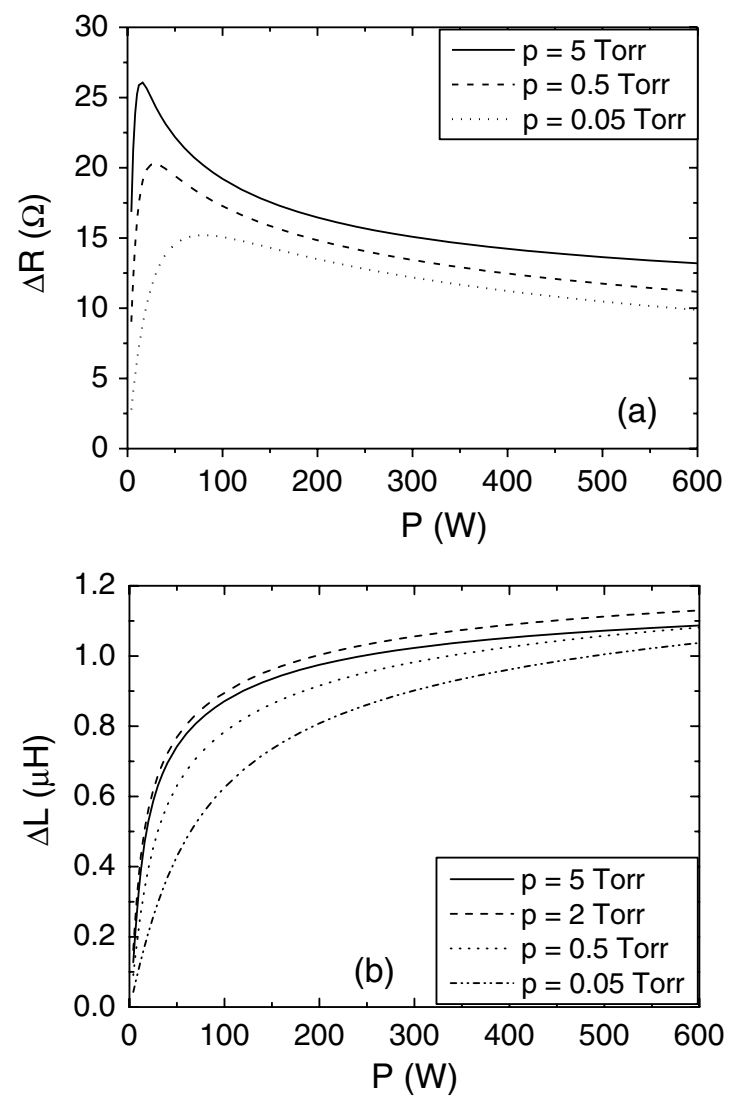

Figure 16. Variation with the absorbed power $P$ of the changes $\Delta R$ in $(a)$ and $\Delta L$ (in $(b))$ of the resistance and the inductance of the primary circuit due to the presence of plasma.

Figure 15 shows also the change of the resistance of the primary circuit due to the variation of the gas-pressure. At low pressures, the increase in the pressure causes a sharp increase in the resistance. At higher pressures the dependence is smoother.

The changes $\Delta R$ of the resistance of the primary circuit with the applied power are shown in figure 16(a). With the $P$-increase (i.e. with the increase in the plasma density), $\Delta R$ first increases, reaches a maximum and then decreases. Such a behaviour is according to the formula

$$
\Delta R=\frac{\omega^{2} M^{2}}{R_{2}^{2}+\omega^{2}\left(L_{2}+L_{\mathrm{e}}\right)^{2}} R_{2},
$$

which could be easily obtained from the transformer presentation (figure 14(a)) of the discharge. With the increase in $P$, the plasma density and, respectively, the plasma conductivity increase, and thus, $R_{2}$ decreases. Therefore, the large values of $R_{2}\left(R_{2}>\omega\left(L_{2}+L_{\mathrm{e}}\right)\right)$ correspond to the range of small $P$-values, where $\Delta R$ increases with $P$ (figure 16(a)), the maximum is at $R_{2}=\omega\left(L_{2}+L_{\mathrm{e}}\right)$ and at high applied power $P, R_{2}$ is small $\left(R_{2}<\omega\left(L_{2}+L_{\mathrm{e}}\right)\right)$ showing that the plasma is an inductive load. This behaviour of $\Delta R$ has been also commented on before $[8,15,48,49]$.

The strong decrease in the skin depth (figure 10(b)) with the increase in the applied power (at low values of the power) causes an increase in the mutual inductance $M$ (higher plasma current radius) and of the plasma inductance $\left(L_{2}\right)$ and, subsequently, an increase in $\Delta L$ (figure $16(b)$ ). 


\section{Conclusions}

A self-consistent fluid-plasma model of inductive discharges in an argon gas is presented in the study. The selfconsistency of the model is based on the generalized Schottky condition employed in the discharge description. The model covers the total range of validity of the diffusion-controlled regime including the transitions to a free-fall regime and to a recombination regime. This is achieved owing to detailed treatment of the electron energy balance, of the nonlinear processes in the charged particle balance and of the momentum equations. With the velocity dependence of the elastic electron-neutral collision frequency taken into account, concepts from the kinetic plasma model are introduced in the fluid-plasma model description of the discharge. The discharge structure is presented based on results for the plasma density, the concentration of the atomic and molecular ions and the population of the metastable states, for the electron temperature and the power absorbed on average by one electron, for the radial distribution of the electric and magnetic fields of the transverse high-frequency wave sustaining the discharge and of the Joule heating and the current density into the plasma. Results for the skin depth and the total plasma current are also given. The modification of the discharge structure with varying gas pressure, applied power and frequency of the field maintaining the discharge is discussed. The changes of the parameters of the external coil due to the presence of the plasma are also obtained.

\section{Acknowledgments}

AS and KhT greatly acknowledge the Alexander-vonHumboldt Foundation for the award, respectively, of a Humboldt prize and a Humboldt Research Fellowship as well as Professor Dr H Schlüter and Professor Dr J Engemann for their kind hospitality. The work is within the EURATOMproject FU06-CT-2003-00139 and project no F-1316 of the National Science Fund in Bulgaria.

\section{References}

[1] Lieberman M A and Lichtenberg A J 1994 Principles of Plasma Discharges and Materials Processing (New York: Wiley)

[2] Hopwood J 1992 Plasma Sources Sci. Technol.1 109

[3] Eckert H U 1962 J. Appl. Phys. 332780

[4] Henriksen B B, Keefer D R and Clarkson M H 1971 J. Appl. Phys. 425460

[5] Eckert H U 1986 Proc. $2^{\text {nd }}$ Annual Int. Conf. on Plasma Chemistry and Technology (San Diego, CA) ed H Boenig (Lancaster, PA: Technomic) p 171

[6] Denneman J W 1990 J. Phys. D: Appl. Phys. 23293

[7] Lister G G and Cox M 1992 Plasma Sources Sci. Technol. 167

[8] Gudmunsson J T and Lieberman M A 1997 Plasma Sources Sci. Technol. 6540

[9] Aliev Yu M, Kaganovich I D and Schlüter H 1997 Phys. Plasmas 42413

[10] Godyak V A and Kolobov V I 1998 Phys. Rev. Lett. 81369

[11] Kortshagen U, Bush C and Tsendin L D 1996 Plasma Sources Sci. Technol. 51

[12] Vasenkov A V and Kushner M J 2002 Phys. Rev. E 66066411
[13] Turner M M 1993 Phys. Rev. Lett. 711844

[14] Ramamurthi B, Economou D J and Kaganovich I D 2003 Plasma Sources Sci. Technol. 12170

[15] Piejak R B, Godyak V A and Alexandrovich B M 1992 Plasma Sources Sci. Technol. 1179

[16] El-Fayoumi I M and Jones I R 1998 Plasma Sources Sci. Technol. 7179

[17] Chabert P, Lichtenberg A J, Lieberman M A and Marakhtanov A M 2001 Plasma Sources Sci. Technol. 10478

[18] Wu Y and Lieberman M A 2000 Plasma Sources Sci. Technol. 9210

[19] Aliev Yu M, Schlüter H and Shivarova A 2000 Guided-Wave-Produced Plasmas (Berlin: Springer)

[20] Makasheva K and Shivarova A 2001 Phys. Plasmas 8836

[21] Berndt J, Makasheva K, Schlüter H and Shivarova A 2002 Plasma Sources Sci. Technol. 11208

[22] Berndt J, Makasheva K, Schlüter H and Shivarova A 2002 J. Appl. Phys. 926461

[23] Schlüter H, Shivarova A and Tarnev Kh 2003 Contrib. Plasma Phys. 43206

[24] Koleva I, Paunska Ts, Schlüter H, Shivarova A and Tarnev Kh 2003 Plasma Sources Sci. Technol. 12597

[25] Koleva I, Makasheva K, Paunska Ts, Schlüter H, Shivarova A and Tarnev Kh 2004 Contrib. Plasma Phys. 44552

[26] Makasheva K, Marinov K, Shivarova A, Stoev L and Tarnev Kh 2004 Vacuum 76369

[27] Lister G G, Li Y-M and Godyak V A 1996 J. Appl. Phys. 798993

[28] Godyak V A, Piejak R B and Alexandrovich B M 1999J. Appl. Phys. 853081

[29] Salabas A, Gousset G and Alves L L 2002 Plasma Sources Sci. Technol. 11448

[30] Shkarovsky I P, Johnston T W and Bachinski M P 1966 The Particle Kinetics of Plasmas (Reading, MA: Adison-Wesley)

[31] Golant V E, Zhilinsky A P and Sakharov I E 1977 Fundamentals of Plasma Physics (Moscow: Atomizdat)

[32] Ramamurthi B, Economou D J and Kaganovich I D 2003 Plasma Sources Sci. Technol. 12302

[33] Shahid R and Kushner M J 1997 J. Appl. Phys. 822805

[34] Jonkers J, Sande M, Sola A, Gamero A, Rodero A and Mullen J 2003 Plasma Sources Sci. Technol. 12464

[35] Böhle A and Kortshagen U 1994 Plasma Sources Sci. Technol. 380

[36] Ferreira C M and Loureiro L 1983 J. Phys. D: Appl. Phys. 161611

[37] Vriens L and Smeets A H M 1980 Phys. Rev. A 22940

[38] Smirnov B M 1978 Physics of Weakly Ionized Gas (Moscow: Nauka)

[39] Bultel A, Ootegem B, Bourdon A and Vervisch P 2002 Phys. Rev. E 65046406

[40] Mehr F J and Biondi M A 1968 Phys. Rev. 176322

[41] Riemann K U 1995 IEEE Trans. Plasma Sci. 23709

[42] Ferreira C M and Loureiro J 1983 J. Phys. D: Appl. Phys. 162471

[43] Makasheva K and Shivarova A 2002 IEEE Trans. Plasma Sci. 30384

[44] Lao C, Gamero A, Sola A, Petrova Ts, Benova E, Petrov G M and Zhelyazkov I 2000 J. Appl. Phys. 877652

[45] Amorim J, Maciel H S and Sudano J P 1991 J. Vac. Sci. Technol. B 9362

[46] Schwabedissen A, Benck E C and Roberts J R 1998 Plasma Sources Sci. Technol. 7119

[47] Marwan H K and Overzet L J 2000 Plasma Sources Sci. Technol. 9545

[48] Kortshagen U, Gibson N D and Lawrer J E 1996 J. Phys. D: Appl. Phys. 291224

[49] Godyak V A, Piejak R B and Alexandrovich B M 1994 Plasma Sources Sci. Technol. 3169 